\title{
Positional Changes of the Hyoid Bone after Correction of the Glosso-Larynx-Hyoid Bone Complex (HBC)
}

\author{
Susumu Mukai \\ Otorhinolaryngology, Mukai Clinic, Kanagawa, Japan \\ Email: s-mukai@da2.so-net.ne.jp
}

How to cite this paper: Mukai, S. (2020) Positional Changes of the Hyoid Bone after Correction of the Glosso-Larynx-Hyoid Bone Complex (HBC). International Journal of Clinical Medicine, 11, 252-256.

https://doi.org/10.4236/ijcm.2020.115026

Received: March 31, 2020

Accepted: May 11, 2020

Published: May 14, 2020

Copyright $\odot 2020$ by author(s) and Scientific Research Publishing Inc. This work is licensed under the Creative Commons Attribution International License (CC BY 4.0).

http://creativecommons.org/licenses/by/4.0/

\begin{abstract}
Human hyoid bone floats in the neck. Downwards of an amount of about half vertebra and $0.3 \mathrm{~cm}$ forward following the correction of the glosso-larynx (CGL) was observed by simple XP. Disappearance of symptoms of the ankyloglossia with deviation of the epiglottis and larynx (ADEL) and increased of body temperature were observed after CGL. The results suggest that hyoid bone makes a complex that controls not only respiration and swallowing but also controls metabolism. It might be called hyoid bone complex (HBC).
\end{abstract}

\section{Keywords}

Respiration, Swallowing, Metabolism, Homo Neanderthalensis

\section{Introduction}

Symptoms and signs of ankyloglossia with deviation of the epiglottis and larynx (ADEL) are caused by respiratory insufficiencies. Correction of the glosso-larynx (CGL) is a surgery for ADEL, consisting in cutting the frontal bundles of genioglossus muscles. Following CGL, the deviated epiglottis and larynx move downwards aligned with the choanae, and improved respiratory rate and pulmonary function and increased body temperature are observed. The possible mechanisms leading to improved respiratory rate and increased body temperature cannot be explained by the cut of the bundles of genioglossus muscle alone and additional factors are likely to play a role, for example positional changes of the hyoid bone occurring after CGL [1] [2] [3] [4] [5].

The aim of this study was to assess the positional changes of the hyoid bone following GCL by using simple X-ray photographs (XPs) in order to assess the possible mechanisms at the level of the hyoid bone that may be related to the 
beneficial effects observed following CGL.

\section{Method}

A total of 232 patients (138 females, 94 males; 17 to 78 years old (yr), average $40.1 \mathrm{yr}$ ) that underwent CGL from 2013 to 2016 were studied by taking XPs before and after CGL (Figure 1).

To assess possible positional changes in the hyoid bone, two measures were extracted from XPs and compared before and after CGL. Specifically, we measured the height of point $\mathrm{V}$ and the length of segment $\mathrm{VH}$, defined in the followings. A horizontal line was drawn to connect the outermost part of the hyoid bone (point $\mathrm{H}$ ) to the vertebrae (point $\mathrm{V}$ ) and the distance between the two points was computed (length of segment $\mathrm{VH}$ ), as shown in Figure 1.

To define the height of point $\mathrm{V}$ along the vertebrae, four possible positions were considered on each vertebra: the upper part (e.g., IIIup), the central part (e.g., III) the lower part (e.g., IIIdn) and the joint between two vertebrae (e.g., III/IV).

The following two measures were assessed and compared before and after CGL:

1) The height of point $V$.

2) The length of segment $\mathrm{VH}$.

For the sake of comparing the length of segment VH before and after CGL, calibrations on the XPs were performed to each film according to the scale on film.

Possible differences in mean height of point $\mathrm{V}$ in the study sample before and after CGL were assessed by using a contingency table test (StatView 4.5) and possible differences in mean length of segment VH before and after CGL were addressed by using Student t-tests (Microsoft Exel Mc2011).

\section{Results}

\subsection{Distribution of Height of Point V}

The height of point $\mathrm{V}$ before CGL ranged from the third vertebra (III) to the

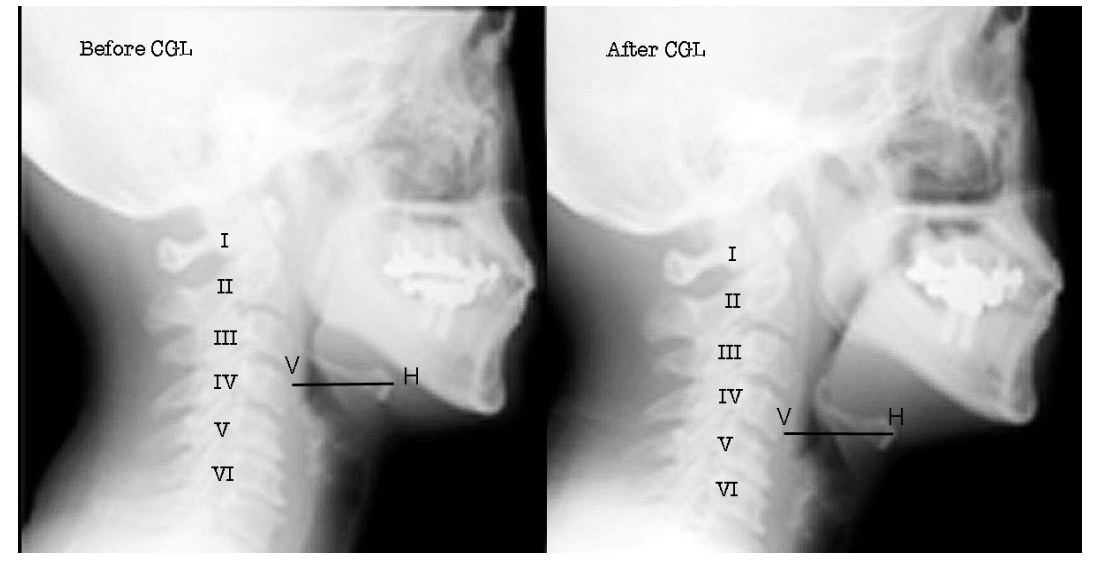

Figure 1. XPs in one of the participants before and after CGL. 
upper part of the fifth vertebra (Vup). After CGL the heights of point V shifted, on average, towards lower vertebrae and ranged from the third (III) to the fifth vertebra $(\mathrm{V})$. The median value of the height of point $\mathrm{V}$ before and after CGL was IV up and IV, respectively, suggesting a trend towards a downward shift (Figure 2).

Statistical analysis showed that there was a significant difference between two distributions $(\mathrm{p}<0.0001)$, suggesting a tendency to a downward shift in the height of point $\mathrm{V}$ following CGL.

\subsection{Length of Segment VH}

The average length of segment VH before and after CGL was $4.4 \mathrm{~cm}$ (s.d. 0.63) and $4.7 \mathrm{~cm}$ (s.d. 0.64), respectively (Female: $4.1 \mathrm{~cm}$ and $4.4 \mathrm{~cm}$; Male: $4.9 \mathrm{~cm}$ and $5.2 \mathrm{~cm}$ ) (Figure 3). There was significant difference between the distribution of length VH before and after CGL $(\mathrm{p}<0.0001)$, suggesting a trend towards an increase in distance between the hyoid bone and the vertebrae following the procedure.

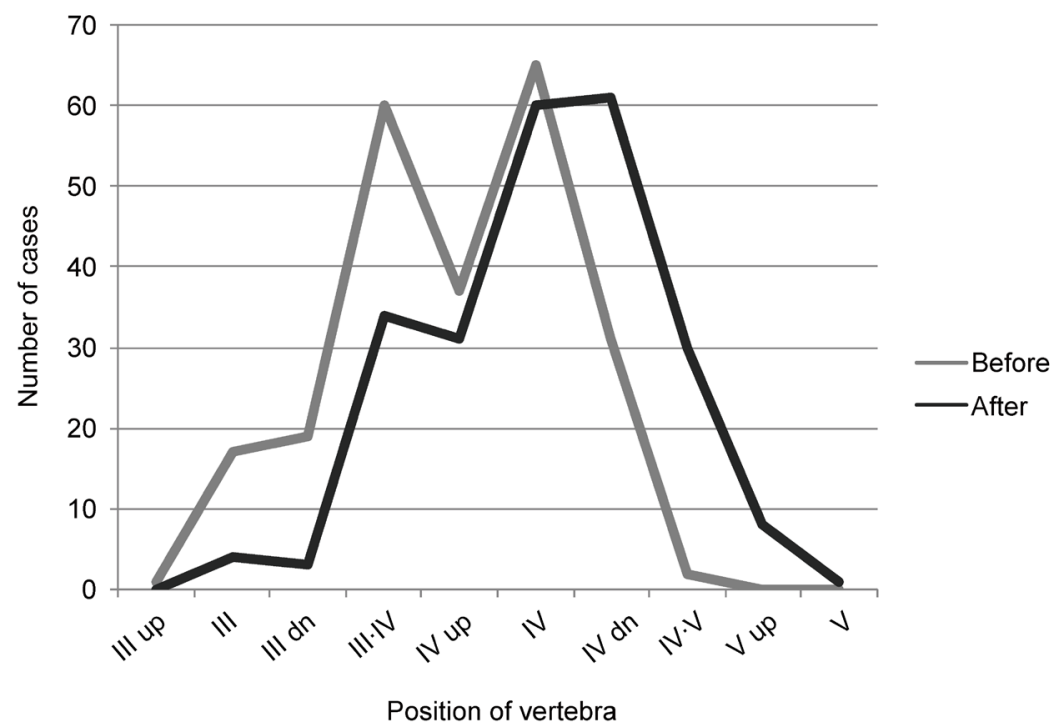

Figure 2. Distribution of the height of point V before and after CGL in the study sample $(\mathrm{N}=232)(\mathrm{p}<0.0001)$.

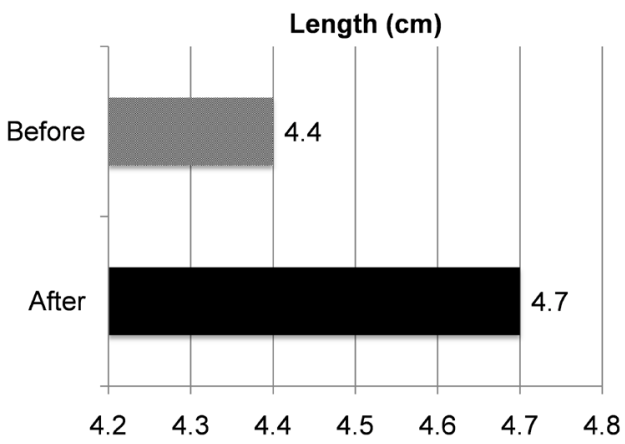

Figure 3. Mean length of segment VH before and after CGL in the study sample $(\mathrm{N}=232)(\mathrm{p}<0.0001)$. 


\section{Discussion}

Phylogenetically, the tongue appeared later. In fishes, the hyoid bones are in the inner margin of the mouth bones [6]. Fishes are able to eat and breathe with the composite of visceral skeleton. They don't need the tongue as mammals.

The tongue muscles are attached at the top of the hyoid bone in humans by the hyoglossal membrane. The tongue muscles are attached to the mandibular bone ventrally. The bottom edge of the hyoid bone the larynx is attached to the thyrohyoid ligament. In turn, the larynx is connected with the trachea, bronchi and lungs. These structures constitute a complex that is called the hyoid bone complex (HBC). The hyoid bone floats in the neck but and the mandibular bone is stable. As the hyoid bone is able to move upwards and downwards via the muscles that are attached to its top and bottom edge, the HBC is able to move upwards and downwards too [7]. As a result, the $\mathrm{HBC}$ in humans is pulled up and forward by the tongue. The CGL is a procedure that cuts the frontal bundles of genioglossus muscles and therefore the $\mathrm{HBC}$ can rotate downward and forward following CGL.

This study showed that the hyoid bone tended to move downwards of about half vertebra and about $0.3 \mathrm{~cm}$ ventrally following CGL. These outcomes may be interpreted in light of the observed improvements following CGL in individuals with ADEL. In fact, disappearance of symptoms and signs of ADEL following CGL (for example, improvement in respiratory function and increase in body temperature) may be related to downward and ventral movements of the hyoid bone that might accelerate respiration [8] [9] [10].

The improvement of symptoms of ADEL and the increase in respiratory function and body temperature observed after CGL may be related to downward and forward movements of the HBC that, in turn, may accelerate physiological functions and may, as such, have further implications in terms of not only respiration and swallowing but also circulation, metabolism, and immune function. The observed improvements are likely to be related to the function of the HBC, a complex structure that regulates respiration and various human functions such as drinking, laughing, singing, swallowing, vomiting, crying, and breathing.

It was reported that the hyoid bone of Homo Neanderthalensis is higher than that of Homo Sapiens [11] [12]. The stylohyoid bone of Homo Neanderthalensis was shorter than that of Homo Sapiens. Therefore the HBC of Homo Neanderthalensis might be shorter and it might have had more limited movements. Related to this, the metabolisms of Homo Neanderthalensis might have been lower than that of Homo Sapiens, possibly contributing to a higher mortality rate, thus suggesting that positional changes of the HBC, such as the ones induced by CGL and demonstrated by this study, may play a role in an evolutionary perspective.

\section{Conclusions}

The tongue pulled HBC upwards. The improvements in symptoms and signs of ADEL observed following CGL as downward and forward movement of the 
hyoid bone could improve respiration and, in turn, contribute to improved physiological function.

There might exist a system that control respiration and swallowing as well as metabolism by the hyoid bone that is hyoid bone complex (HBC).

\section{Consent}

All included patients provided their written informed consent, and the study's protocol was approved by the research ethics committee of Yamato City Medical Association.

\section{Conflicts of Interest}

The author declares no conflicts of interest regarding the publication of this paper.

\section{References}

[1] Mukai, S., Mukai, C. and Asaoka, K. (1991) Ankyloglossia with Deviation of the Epiglottis and Larynx. Annals of Otology, Rhinology \& Laryngology, 100, 1-20. https://doi.org/10.1177/00034894911000S501

[2] Mukai, S. and Mukai, C. (1991) New Disease with Ankyloglossia and Dislocation of the Epiglottis and of the Larynx-Dyspnea of the Newborn and Suckling Infants. In: Takishima, H., Ed., Advances in the Biosciences, Pergamon Press, New York, 191-193.

[3] Mukai, S., Mukai, C. and Asaoka, K. (1993) Congenital Ankyloglossia with Deviation of the Epiglottis and Larynx: Symptoms and Respiratory Function in Adults. Annals of Otology, Rhinology \& Laryngology, 102, 620-624. https://doi.org/10.1177/000348949310200810

[4] Nitta, M., Mukai, S. and Mukai, C. (2000) Locational Changes between Hyoid Bone and Cranium after Correction of Glosso-Larynx (CGL) (Japanese with English Abstract). Practica Otologica (Kyoto), 93, 43-47. https://doi.org/10.5631/jibirin.93.43

[5] Mukai, S. (2018) Positional Changes in the Hyoid Bone, Hypopharynx, Cervical Vertebrae, and Cranium before and after Correction of Glosso-Larynx (CGL). Archives of Otolaryngol and Rhinology, 4, 37-43. https://doi.org/10.17352/2455-1759.000073

[6] Kent, G.C. and Carr, R.K. (2015) Comparative Anatomy of the Vertebrates. 9th Edition, Midori Shoboh Ltd., Tokyo.

[7] Mukai, S. (2018) The Hyoid Bone Complex. Zetuyuchaksho Kenkyuukai Kaiho, 12-21. (In Japanese)

[8] Mukai, S. (2018) Increase of Body Temperature after Correction of Glosso-Larynx and Expansion of Vesibular Oris. Annals ADEL, Nihon Zetuyuchakusho Gakkaishi, 22, 1-4. (In Japanese)

[9] Mukai, S. (2018) Increase of Body Temparature after Correction of the Glosso-Larynx (CGL) and Expansion of the Vestibular Oris (EVO). Am J Otolaryngology and Head and Neck Surg, 1, 1-3.

[10] Mukai, S. (2019) Changes of the Hyoid Bone after Correctio of the Glosso-Larynx. British Journal of Otolaryngology, 1, 104-106.

[11] Lieberman, D.E. (2011) The Evolution of Human Head. The Belknap Press of Harverd Press, Cambridge.

[12] Lieberman, D.E. (2014) The Story of Human Body. Vintage Books, New York. 